\title{
The application of early ostomy closure on the infants with necrotizing enterocolitis
}

\author{
Ji-xue Zhao ${ }^{1, a}$, Chuan Zhang ${ }^{1}$, Xue-song Zhao ${ }^{1}$, Chun-yu Dong ${ }^{1}$, and Xin Fu ${ }^{2, b}$ \\ ${ }^{1}$ Department of Pediatric Surgery, The First Hospital of Jilin University, Changchun 130021, China; \\ ${ }^{2}$ Nursing Administration Department, China-Japan Union Hospital of Jilin University, Changchun \\ 130033, China

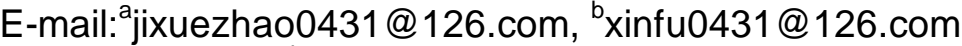 \\ ${ }^{b}$ corresponding author
}

\begin{abstract}
Keywords: Necrotizing enterocolitis, ileostomy, ostomy closure.
\end{abstract}
Abstract. Objective: To explore the application effect of early ostomy closure on the patient with necrotizing enterocolitis (NEC). Methods: From January 2012 to March 2015, 23 infants with NEC which had been performed ileostomy were treated with early ostomy closure and normal ostomy closure in the First hospital of Jilin University. These infants were divided into two groups. In control group, from January 2012 to June 2013, 11 patients were performed normal ostomy closure(3-6 months after ostomy), In experimental group, from July 2013 to March 2015, 12 patients were performed early ostomy closure(less 3 months after ostomy), clinical data were retrospectively analyzed, including the time from ostomy to ostomy closure, the mean operation time, the intraoperative bleeding, the averaged length of stay (ALS), the incidence of postoperative wound infections. Results: The time from ostomy to ostomy closure of experimental group was obviously less than that of control group[ $(1.49 \pm 0.64)$ months vs $(4.43 \pm 1.23)$ months] $(\mathrm{P}<0.05)$. There were no significant difference in the mean operation time between the two groups[ ( 62.4 \pm 9.3 ) minutes vs $(64.5 \pm 10.7)$ minutes $](\mathrm{P}>0.05)$. The difference of the operative blood loss in two groups were not statistically significant $[(6.2 \pm 1.6) \mathrm{ml}$ vs $(5.5 \pm 1.4) \mathrm{ml}](\mathrm{P}>0.05)$. The difference of the averaged length of stay (ALS) in two groups were not statistically significant [( 7.5 \pm 1.5$) \mathrm{d}$ vs $(7.3 \pm 1.1) \mathrm{d}](\mathrm{P}>0.05)$, and the incidence of postoperative wound infections were not different between two groups too ( $16.7 \%$ vs18.2\% ) ( $>>0.05)$. Conclusions: The early ostomy closure did not have the negative effect for the infants with NEC. Compared with normal ostomy, early ostomy closure could restore the bowel function earlier, and could avoid Long-term dehydration and malnutrition during the waiting for ostomy closure.

\section{Introduction}

Necrotizing enterocolitis(NEC) is one of the serious disease which endanger the neonatal life. Case fatality rate is as high as $10 \%-50 \%$. Although many scholars had done many research about NEC, the case fatality rate did not decrease [1]. And the data show that $20 \%-60 \%$ of the children still need the surgery[2]. NEC can affects the small intestine and colon, but the predilection site is in the distal ileum and ascending colon proximal. So the operation of NEC always include the resection of necrotic bowel and ostomy of the distal ileum, and 3-6 months after the ostomy, ostomy closure will be performed. Because of the loss of fluid and electrolytes and nutrition malabsorption after the ostomy[3], many children always get severe malnutrition, and need intermittent parenteral nutrition to support the life. Even many of them need hospitalization to correct dehydration and malnutrition, which will increase the children's suffering and burden of the parents. If we can perform the early ostomy closure which will restore the intestinal function has become our main research topic. Through the retrospective analysis, Struijs MC pointed out, there is no evidence that postoperative complications is different between the early ostomy closure and late ostomy closure[4]. Based on these considerations above, 
From August of 2013, we try to regulate the timing of ostomy closure to less than 3 months from more than 3 months, and we have achived desirable effect.

\section{Patients and methoths}

Patients. From January of 2012 to March of 2015, 23 infants with NEC which had been performed ileostomy in the neonatology department, the First Hospital of Jilin University were enrolled in this retrospective analysis. Each patient signed an informed consent form. Approval was obtained from the institutional review committee of Jilin University.

\section{Study Design}

The patients were divided into two groups according to the surgical methods: normal ostomy closure group (control group): from January 2012 to June 2013, 11 patients were performed normal ostomy closure(3-6 months after ostomy); early ostomy closure group(experimental group): from July 2013 to March 2015, 12 patients were performed early ostomy closure(less 3 months after ostomy).

Normal ostomy closure (before reform): According to the traditional methods, we can perform the ostomy closure 3-6 months after the first operation.

Early ostomy closure (after reform): We can perform the the ostomy closure less 3 months after ostomy. During two weeks before the ostomy closure, we should treat the patients with intravenous hyperlimentation and proper enteral nutrition to correct dehydration, ion disorder and malnutrition. And when the general condition improved, we can perform ostomy closure timely.

\section{Evaluation Criteria of Treatment Effects}

Evaluation criteria included the time from ostomy to ostomy closure, the intraoperative bleeding, the mean operation time, the averaged length of stay (ALS), the incidence of postoperative wound infections.

\section{Statistical Analysis}

All measured parameters including the time from ostomy to ostomy closure, the mean operation time, the operative blood loss, the averaged length of stay (ALS), were analyzed by the statistical software program Statistical Product and Service Solutions (SPSS) 17.0 (SPSS Inc., Chicago, IL, USA) and expressed as mean \pm standard deviation $( \pm \mathrm{s})$, and t-test was used. Enumeration data including gender, prevalence frequency were analyzed by $\chi 2$ test. $\mathrm{P}<0.05$ was considered significant.

\section{Results}

\section{Baseline Characteristics}

There were no significant difference $(\mathrm{P}>0.05)$ in general data including first operation age, gender between the two groups (Table 1.).

Table 1. Baseline characteristics of study patients

\begin{tabular}{cccc}
\hline Characteristic & control group(11 cases) & experimental group (12 cases) & $P$ value \\
\hline First operation age (days) & $8.6 \pm 2.6$ & $9.0 \pm 3.1$ & non-significant \\
Female & 4 & 5 & non-significant \\
Male & 7 & 7 & non-significant \\
\hline
\end{tabular}

\section{Evaluation of Treatment Effects}

All affected infants have undergone ostomy closure successfully and have recovered satisfactorily. No dead case was found during the hospitalization.

The time from ostomy to ostomy closure, mean operation time, the operative blood loss, the averaged length of stay (ALS), the incidence of postoperative wound infections after operation were analyzed. The time from ostomy to ostomy closure of experimental group was obviously less than that of control group $(\mathrm{P}<0.05)$. There were no significant difference in the mean operation time between the two groups $(\mathrm{P}>0.05)$. The difference of the operative blood loss in two groups were not statistically significant $(\mathrm{P}>0.05)$. The difference of the averaged length of stay (ALS) in two groups were not statistically significant $(\mathrm{P}>0.05)$. There were no significant difference in the incidence of postoperative 
wound infections between the two groups $(\mathrm{P}>0.05)$, as is shown in Table 2. Postoperative follow-up was conducted for 3-6 months.

Table 2 Efficacy of the two groups

\begin{tabular}{lccl}
\hline & control group(11 cases) & experimental group(12 cases) & $P$ value \\
\hline Ostomy closure time (months) & $4.43 \pm 1.23$ & $1.49 \pm 0.64$ & $P<0.05$ \\
Mean operation time(minutes) & $64.5 \pm 10.7$ & $62.4 \pm 9.3$ & non-significant \\
Blood loss (ml) & $5.5 \pm 1.4$ & $6.2 \pm 1.6$ & non-significant \\
The averaged LOS *(days) & $7.3 \pm 1.1$ & $7.5 \pm 1.5$ & non-significant \\
Infection incident & $2 / 11$ & $2 / 12$ & non-significant \\
\hline
\end{tabular}

* LOS: length of stay

\section{Discussion}

NEC is one of the most serious and common diseases of the neonatal period, and a familiar surgery urgent abdominal disease. NEC can cause severe multiple organ and systemic inflammation reaction, and always lead to the mortality rate of 20\%-30\%[5-7]. The survivor always accompanied enterostenosis(15\%-30\%), short bowel syndrome and cholestatic liver diseases, and so on[8]. NEC is very hard to treat, and most of the infants with NEC need surgery, and the early surgery is safe and effective[9].

NEC can affects the whole small intestine and colon, but the predilection site is in the distal ileum and ascending colon proximal. So the operation of NEC always include the resection of necrotic bowel and ostomy of the distal ileum, and after 3-6 months, ostomy closure will be performed. Because the necrotic bowel loops has been removed, the left loops have become very short(always less than $100 \mathrm{~cm}$ and even only left $40-50 \mathrm{~cm}$ ), which will obviously decrease the nutrition absorption, and lead to severe malnutrition. Additionally, ileostomy cause that the water cannot be absorbed by colon, which will lead to the great loss of fluid and electrolyte. Many of the patients suffered from frequent diarrhea and subsequent severe dehydration. Longstanding dehydration and malnutrition can lead to the difficult recovery, which can cause that the infants cannot tolerate the surgery, and even can endanger their lives. Most of the patients always need intermittent parenteral nutrition to support the life. While the long time parenteral nutrition can lead to many complications such as liver damage, this will set up a circle of aggravating. Many patients need the repeated hospitalization to correct the severe dehydration and malnutrition, which can increase not only the patients'suffering, but also the economic burden of parents. Therefore, we positively discuss the possibility of early ostomy closure.

Ostomy formation is inevitable in certain cases, and almost half of the patients were operated on for necrotizing enterocolitis (NEC) [10]. Ostomy can cause many ostomy-related complications, such as stricture, parastomal hernia, prolapse, wound infection, wound fistula, wound dehiscence, and small bowel obstruction [11-13]. So performing the early ostomy closure and recover the normal gut function seems imperative. And at the same time, the subsequent ostomy closure can also carry lots of complications, including wound infection, wound dehiscence, enterocutaneous fistula, bowel obstruction, anastomotic leak, and anastomotic stricture $[3,11]$. Therefore, it is uncertain whether it is safe to perform early ostomy closure, and what is the optimal timing of ostomy closure.

Successful ostomy closure with as less as possible complications depend on quite a number of factors[14], the most important thing is the chosing of operation time. The infants'ostomy was always temporary ostomy, and had more complications than that of adults. Additionally, because of the nursing difficulty and the pressure of family and society, more and more parents want the early ostomy to recover the normal life, and the early ostomy also can reduce the complications[15]. However, there exist severe and extensive inflammatory reaction around the stroma during the early phase of the ostomy, early ostomy closure always lead to the more complications and even the treatment failure. So there was no final conclusion about the optimal timing of ostomy closure after the ileostomy. In the ideal state, we should perform the ostomy closure after the relief of protopathy and the absorption of celiac inflammation. While for the infants with NEC, the position of ostomy was always on the distal ileum and even the higher location, which will lead to severe electrolytes and fluid imbalance[3]. In the 
face of this, strong postoperative anti-inflammatory and support treatment is necessary, when the condition recover, we should perform the ostomy timely. Struijs MC, et al.pointed out, after stabilization of the patient, ostomy closure can be considered within 6 weeks during the same admission as the initial laparotomy[16].

Early closure is associated with several advantages. For one, maintaining a normal fluid and electrolyte balance is best helped by restoration of enterocolonic continuity as soon as possible. Another advantage of early ostomy closure was the possible prevention of distal strictures. The observed rate of distal strictures after ostomy formation was around $40 \%$ [11]. Early closure of the ostomy might lead to fewer strictures caused by feedings[4]. Al-Hudhaif et al[17]. found a longer duration to achieve full enteral nutrition in the EC group (19.1 days in the EC group vs. 7.2 days in the LC group), so it is controversial whether we should perform the ostomy closure early or not. At present, because many surgeons worry about the severe postoperative abdominal adhesions and the morbidity associated with ventilation anticipated in case of earlier closure, they always choose to perform the ostomy closure at least 8 weeks after ostomy or until the infant weighs $2 \mathrm{~kg}[18-20]$. Yin Qiang,et al[21]. point out it is safe to perform the ostomy closure more than 3 months after the ostomy. Another articles also indicate when the patients' condition is not so good and the body has not revovered, we should not perform the ostomy closure hurriedly. As a general rule, we can perform it 3-6 months after the ostomy.

At present, most of the doctors always perform the ostomy closure 3-6 months after the ostomy. Through the retrospective analysis, Struijs MC pointed out, this systematic review showed that complication rate did not differ between early and late closure of ostomy in patients with necrotising enterocolitis[4].

According to the above analysis, we try to regulate the time of ostomy closure to less than 3 months from more than 3 months. From the retrospective analysis of the clinical data, we can find that the time from ostomy to ostomy closure of experimental group was obviously less than that of control group. That indicate that we do perform the ostomy closure earlier. There were no significant difference in the mean operation time between the two groups. The difference of the operative blood loss in two groups were not statistically significant. The difference of the averaged length of stay (ALS) in two groups were not statistically significant and the incidence of postoperative wound infections were not different between two groups too. And those indicate that early ostomy closure did not have a negative effect for the infants. While the early ostomy closure can recover the gut function early, avoid the dilemma of long time dehydration and malnutrition during the waiting for ostomy closure, and the advantages are obvious. So we think that early ostomy closure is clinically feasible.

In our experience, as for the early ostomy closure, the most important of all is to assess and restore the nutrition state of the infants with NEC before the ostomy closure. During two weeks before the ostomy closure, according to the different condition of the different patients, including the weight, the age, the degree of dehydration and malnutrition, and so on, we should treat the patients with intravenous hyperlimentation and proper enteral nutrition to correct dehydration, ion disorder and malnutrition. And when the general condition improved, we can perform ostomy closure timely.

In conclusion, currently no final conclusion has yet been reached on the time from ostomy to ostomy closure. Under the condition of ensuring the safety of patients, we reduce the waiting time properly in accordance with specific conditions, and achieved a good clinical effect.

\section{Conclusions}

The early ostomy closure did not have the negative effect for the infants with NEC and it could restore the bowel function earlier, avoid long-term dehydration and malnutrition during the waiting for the ostomy closure. And the early ostomy closure is clinically feasible for the infants with NEC which had been performed ileostomy..

\section{References}

[1] Yost CC . Neonatal necrotizing enterocolitis: diagnosis, management, and pathogenesis[J] . J Infus Nurs , 2005 , 28 ( 2) :130-134. 
[2] Thompson AM, Bizzarro MJ. Necrotizing enterocolitis in newborns:pathogenesis, prevention and management. Drugs. 2008;68(9):1227-1238.

[3] Steinau G , Ruhl KM , Hörnchen H, et al . Enterostomy complications in infancy and childhood[J] . Langenbecks Arch Surg , 2001 , 386(5) : 346-349 .

[4] Struijs MC, Sloots CE, Hop WC, et al. The timing of ostomy closure in infants with necrotizing enterocolitis: a systematic review[J]. Pediatr Surg Int. 2012 Jul;28(7):667-672.

[5] Thompson A, Bizzarro M, Yu S , et al. Risk factors for necrotizing enterocolitis totalis: a case-control study [J]. J Perinatol , 2011,31 (11) : 730-738.

[6] Gephart SM , McGrath JM , Effken JA, et al. Necrotizing enterocolitis risk: state of the science[J]. Adv Neonatal Care , 2012 , 12 (2) : 77-87.

[7] Diehl Jones ML, Askin DF, Nutritional modulation of neonatal outcome[J],AACN Clinical Issues, 2004,15(1):83-96.

[8] Neu J, Chen M, Beierle E. Intestinal innate immunity: how does it relate to the pathogenesis of necrotizing genterocolitis[J]. Semin Pediatr Surg, 2005,14(3):137-144.

[9] Gfroerer S, Fiegel H, Schloesser RL, et al. Primary laparotomy is effective and safe in the treatment of necrotizing enterocolitis[J]. World J Surg. 2014, 38(10):2730-2734.

[10] Eltayeb AA, Mostafa MM, Ibrahim NH et al. The role of surgery in management of necrotizing enterocolitis[J]. Int J Surg, 2010,8:458-461.

[11] O'Connor A, Sawin RS. High morbidity of enterostomy and its closure in premature infants with necrotizing entercolitis[J]. Arch Surg. 1998,133:875-880.

[12] Aguayo P, Fraser JD, Sharp S, et al. Stomal complications in the newborn with necrotizing enterocolitis[J]. J Surg Res,2009,157:275-278.

[13] Haberlik A, Hollwarth ME, Windhager U, et al.Problems of ileostomy in necrotizing enterocolitis. Acta Paediatr Suppl, 1994, 396:74-76.

[14] Bischoff A, Levitt MA, Lawal TA , Peña A . Colostomy closure:how to avoid complications [ J ] . Pediatr Surg Int , 2010 , 26( 11):1087-1092 .

[15] Cigdem MK, Onen A, Duran H, et al. The mechanical complications of colostomy in infants and children: analysis of 473 cases of a single center [ J ] . Pediatr Surg Int , $2006,22(8)$ :671-676.

[16] Struijs MC, Poley MJ, Meeussen CJ, et.al. Late vs early ostomy closure for necrotizing enterocolitis: analysis of adhesion formation, resource consumption, and costs. J Pediatr Surg, 2012, 47 (4): 658-664.

[17] Al-Hudhaif J, Phillips S, Gholum S et al .The timing of enterostomy reversal after necrotizing enterocolitis. J Pediatr Surg, 2009, 44:924-927.

[18] Kinouchi K. Anaesthetic considerations for the management of very low and extremely low birth weight infants[J]. Best Pract Res Clin Anaesthesiol.2004, 18: 273-290.

[19] Holzman RS . Morbidity and mortality in pediatric anesthesia[J]. Pediatr Clin North Am. 1994, 41:239-256.

[20] Rappaport B, Mellon D, Simone A, et al. Defining safe use of anesthesia in children. N Engl J Med, 2011, 364:1387-1390.

[21] YIN Qiang, ZHOU Xiaoyu , XIAO Ya ling. Clinical analysis on 425 cases of enterostomy in children[J]. Chinese Journal of General Surgery. 2008. 04(17): 372-375. 